\title{
DETERMINATION OF STRESSES CAUSED BY INFINITELY LONG LINE LOADS ON SEMI-INFINITE ELASTIC SOILS USING FOURIER TRANSFORM METHOD
}

\author{
H. N. Onah ${ }^{1}$, N. N. Osadebe ${ }^{2}$, C. C. Ike ${ }^{3,}$ and C. U. Nwoji ${ }^{4}$ \\ 1, 2, 4 DEPT OF CiVIL ENGINEERING, UNIVERSITY OF NigERIA, NSUKKA. ENUGU STATE. NIGERIA

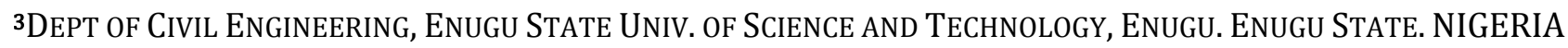 \\ E-mail addresses:1hyginus.onah@unn.edu.ng,2mkemamaka@yahoo.co.uk,3ikecc2007@yahoo.com \\ ${ }^{4}$ clifford.nwoji@unn.edu.ng
}

\begin{abstract}
In this work, the Fourier transform method has been applied to the determination of stresses induced by infinitely long line loads on semi-infinite homogeneous elastic soils. Airy's stress functions of the Cartesian coordinates system were used to express the governing equations of plane strain elasticity for a semi-infinite homogeneous soil as a biharmonic problem. The fourth order partial differential equation was then solved by an exponential Fourier transform technique, with respect to the space valuable $x$ where $-\infty \leq x \leq \infty$; and the resulting solutions made subject to the stress - boundary conditions. The stresses obtained were found to be exactly identical with solutions obtained by integrating Boussinesq's solutions for a point load which are available in the technical literature. The stresses determined in the present study were also exactly identical with the Flamant's solution for the same problem; obtained by assuming a stress function in terms of the cylindrical coordinates.
\end{abstract}

Keywords: Fourier transform method, Airy's stress function, plane strain elasticity, line load, biharmonic problems.

\section{INTRODUCTION}

The determination of the distribution of stresses in semi-infinite elastic soils due to distributed loads on the boundary is a problem of the theory of elasticity. Consequently, solutions are obtained by the simultaneous application of the requirements for differential equations of equilibrium of an infinitesimal element, the stress-strain laws and the strain-displacement equations [1]. There are two basic approaches used in solving this problem in general, namely: stress-based approach and displacement based approach.

Analytical closed form expressions for the stress fields induced in a semi-infinite elastic soil by a line load of infinite extent acting normal to the plane surface have been obtained by Flamant and presented in the technical literature [2 - 4]. Analytical expressions for the stress fields can also be obtained by integrating Boussinesq's solutions for the point load applied on the surface of a semi-infinite linear elastic soil.
Solutions originally obtained by Cummings [5] and Gray [6] have been presented by Timoshenko [7], New mark [8], Hall [14] and Forster and Fergus [10].

Flamant considered the problem of a vertical line load on a homogeneous, isotropic, linear elastic soil half space as the two dimensional equivalent of Boussinesq's point load problem. He then considered it as the superposition of an infinite number of point loads uniformly distributed along the $y$ axis as shown in Figure 1. He obtained the stresses in the $x z$ plane as: $[11,12]$

$$
\begin{array}{r}
\sigma_{z z}=\frac{2 p}{\pi} \frac{z^{3}}{r^{4}}=\frac{2 p}{\pi r} \cos ^{3} \theta \\
\sigma_{x x}=\frac{2 p}{\pi} \frac{x^{3}}{r^{4}}=\frac{2 p}{\pi r} \cos ^{2} \theta \\
\sigma_{x z}=\tau_{x z} \frac{2 p}{\pi} \frac{x z^{2}}{r^{4}}=\frac{2 p}{\pi r} \cos ^{2} \theta
\end{array}
$$

On the basis of Flamant's solutions, many other solutions for stresses due to distributed strip loads as shown in Figure 2 have been obtained using the principle of superposition. An example is the case of 
uniform load of intensity $p$ on a strip of width $2 a$ [13], [3], for which the stresses are:

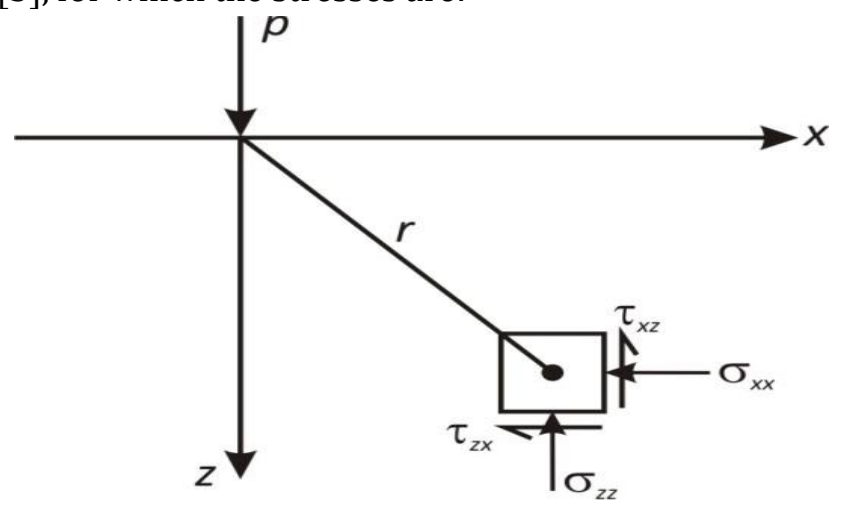

Figure 1: Flamant's problems

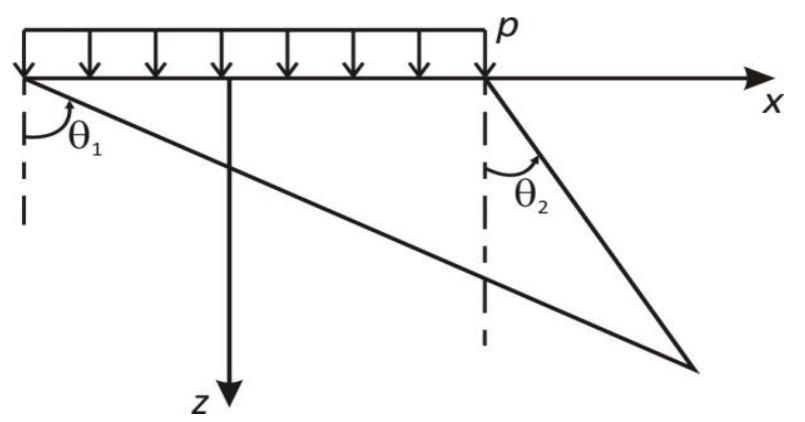

Figure 2: Strip load on an elastic half space soil

$$
\begin{gathered}
\sigma_{z z}=\frac{p}{\pi}\left[\left(\theta_{1}-\theta_{2}\right)+\sin \theta_{1} \cos \theta_{1}-\sin \theta_{2} \cos \theta_{2}\right] \\
\sigma_{x x}=\frac{p}{\pi}\left[\left(\theta_{1}-\theta_{2}\right)-\sin \theta_{1} \cos \theta_{1}+\sin \theta_{2} \cos \theta_{2}\right] \\
\tau_{x z}=\frac{p}{\pi}\left(\cos ^{2} \theta_{2}-\cos ^{2} \theta_{1}\right)
\end{gathered}
$$

In the centre of the plane for $x=0, \theta_{2}=-\theta_{1}$ and the stresses become:

$$
\begin{aligned}
\sigma_{z z}(x=0) & =\frac{2 p}{\pi}\left(\theta_{1}+\sin \theta_{1} \cos \theta_{1}\right) \\
\sigma_{x x}(x=0) & =\frac{2 p}{\pi}\left(\theta_{1}+\sin \theta_{1} \cos \theta_{1}\right) \\
\tau_{x z}(x=0) & =0
\end{aligned}
$$

The variations of the stresses $\sigma_{x x}$ and $\sigma_{z z}$ with depth are shown in Figure 2.

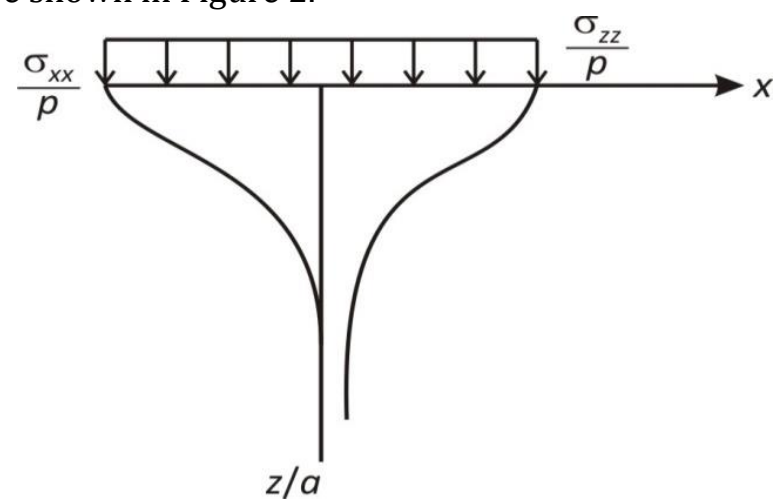

Figure 3: Distribution of $\sigma_{x x}$ and $\sigma_{z z}$ on the centre of a strip footing
Both stresses $\sigma_{x x}$ and $\sigma_{z z}$ tend to zero as the depth increases $(z \rightarrow \infty)$, but the horizontal normal stress tends towards zero much faster than the vertical normal stress $[6,13]$. The elastic stress distribution on a typical retaining wall as shown in Figure 4 is another important application of stress analysis, as presented in Verruijt [3].

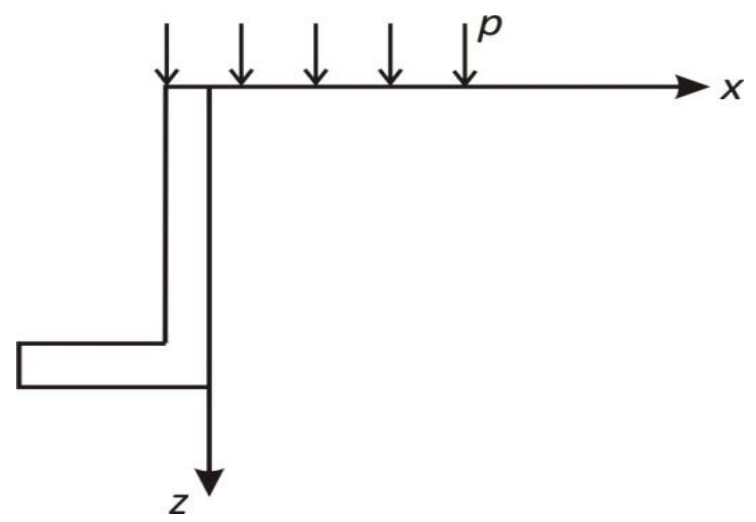

Figure 4: Strip load next to a smooth rigid wall

The stresses are, from [3]:

$\sigma_{z z}(x=0, z)=\frac{2 p}{\pi}\left(\tan ^{-1} \frac{a}{z}+\frac{a z}{a^{2}+z^{2}}\right)$
$\sigma_{X X}(x=0, z)=\frac{2 p}{\pi}\left(\tan ^{-1} \frac{a}{z}+\frac{a z}{a^{2}+z^{2}}\right)$

The total elastic lateral force $P_{e}$, on a wall of height $H$ is:

$$
\begin{gathered}
P_{e}=\int_{0}^{H} \sigma_{x x}(Z) d z \\
P_{e}=\frac{2}{\pi} p H \tan ^{-1} \frac{a}{H}
\end{gathered}
$$

For a very deep wall, $\mathrm{H}>>\mathrm{a}$, then:

$$
\begin{aligned}
& \tan ^{-1} \frac{a}{H} \approx \frac{a}{H} \\
& P_{e}=\frac{2}{\pi} p a=0.637 p a
\end{aligned}
$$

For a very shallow wall, $\mathrm{H}<<\mathrm{a}$, then

$$
\begin{aligned}
& \tan ^{-1}\left(\frac{a}{H}\right) \approx \frac{\pi}{2} \\
& \text { Then } P_{e}=p H
\end{aligned}
$$

\section{GOVERNING EQUATIONS}

The equations governing the determination of stress fields in a loaded linear elastic soil of semi-infinite extent are derived from considerations of the differential equations of equilibrium, the straindisplacement relationships (kinematic considerations), stress-strain laws and straincompatibility relations [1]. The differential equations 
of equilibrium are given in the absence of body forces as

$$
\begin{aligned}
& \frac{\partial \sigma_{x x}}{\partial \sigma}+\frac{\partial \sigma_{x z}}{\partial z}=0 \\
& \frac{\partial \sigma_{x z}}{\partial x}+\frac{\partial \sigma_{z z}}{\partial z}=0
\end{aligned}
$$

In (18) and (19), $\sigma_{x x}, \sigma_{z z}$ are normal stresses, $\tau_{x z}$ is the shear stress, and $x, z$ are Cartesian coordinate axes.

The strain-displacement equations, derived from geometrical considerations, for linear infinitesimal behaviour are:

$\varepsilon_{x x}=\frac{\partial u}{\partial x}, \varepsilon_{z z}=\frac{\partial w}{\partial z} \quad y_{x z}=\frac{\partial u}{\partial z}, \varepsilon_{z z}=\frac{\partial w}{\partial x}$

where $\varepsilon_{x x}, \varepsilon_{z z}$ are normal strains, $\gamma_{x z}$ is the shear strain, and $u, w$ are displacement components in the $x$ and $z$ coordinate directions respectively.

The plane strain conditions are applied (assumed) as:

$$
\varepsilon_{\gamma \gamma}=\gamma_{\gamma z}=\gamma_{\gamma x}=0
$$

where $\varepsilon_{y y}$ is the normal strain and $\gamma_{y z}$ and $\gamma_{y x}$ are shear strains and this implies that

$$
\begin{aligned}
\tau_{z} & =\tau_{\gamma x}=0 \\
\sigma_{y y} & =\mu\left(\sigma_{x x}+\sigma_{z z}\right)
\end{aligned}
$$

where $\mu$ is the Poisson's ratio, $\tau_{y z}$ and $\tau_{y x}$ are shear stresses on the $y z$ and $y x$ coordinate planes, respectively.

The stress-strain laws for plane strain conditions are

$$
\begin{gathered}
\varepsilon_{x}=\frac{1}{E}\left(1-\mu^{2}\right) \sigma_{x x}-\mu(1-\mu) \sigma_{z z} \\
\varepsilon_{z}=\frac{1}{E}\left(1-\mu^{2}\right) \sigma_{z z}-\mu\left(1-\mu^{2}\right) \sigma_{x x} \\
\gamma_{x z}=\frac{1}{G} \tau_{x z}=\frac{2(1+\mu)}{E} \tau_{x z}
\end{gathered}
$$

Where $E$ and $G$ are called the modulus of elasticity and the shear modulus, respectively. The three in plane strains were obtained from two displacements and are not completely independent of each other. The strain compatibility equation is given as:

$$
\frac{\partial^{2} \varepsilon_{x x}}{\partial z^{2}}+\frac{\partial^{2} \varepsilon_{z z}}{\partial x^{2}}=\frac{\partial^{2} \gamma_{x z}}{\partial x \partial z}
$$

The governing equations of plane strain elasticity given in Equations (8) - (26) are statically indeterminate since there are three stress components $\sigma_{x x}, \sigma_{z z}$ and $\tau_{x z}$ and only two equations of equilibrium. Hence, we need to impose the additional constraint that the strains determined from the stressstrain law are compatible. Compatibility is ensured by defining stress functions that satisfy the plane-strain elasticity problem, in this case. Airy's stress function $\phi(x, z)$ is derived such that the stress fields are obtained as:

$$
\sigma_{x}=\frac{\partial^{2} \phi}{\partial z^{2}} \quad \sigma_{z}=\frac{\partial^{2} \phi}{\partial x^{2}} \quad \tau_{x z}=-\frac{\partial^{2} \phi}{\partial x \partial z}
$$

Airy's stress functions can be shown to satisfy the stress compatibility condition if:

$$
\begin{aligned}
& \nabla^{4} \phi=\nabla^{2} \nabla^{2} \phi=0 \\
& \text { where } \nabla^{4}=\frac{\partial^{4}}{\partial x^{4}}+2 \frac{\partial^{4}}{\partial x^{2} \partial \mathrm{z}^{2}}+\frac{\partial^{4}}{\partial z^{4}}
\end{aligned}
$$

$\nabla^{4}$ is the biharmonic operator, while $\nabla^{2}$ is the two dimensional Laplacian operator.

\section{METHODOLOGY AND RESULTS}

Consider a line load on a semi-infinite elastic soil with the coordinate axes defined as shown in Figure 5.

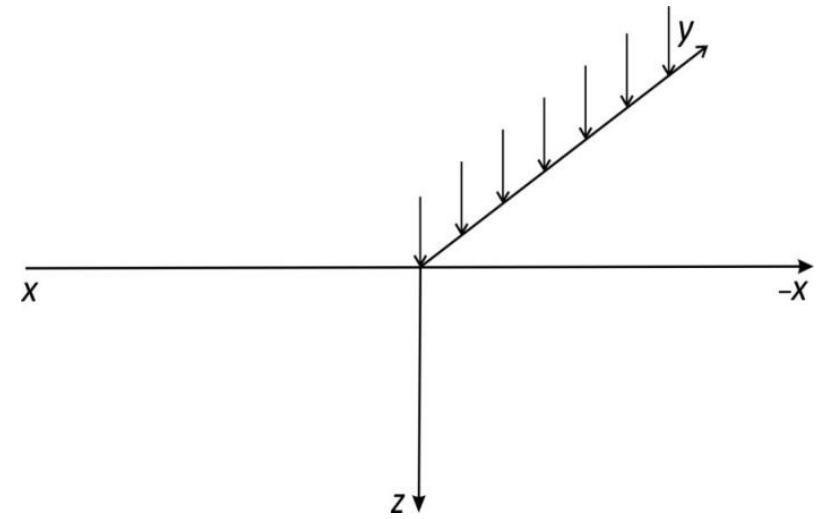

Figure5: Line load of uniform intensity on a semi-infinite elastic soil

For plane strain deformation, the displacement field is $u_{x}=u(x, z), \quad u_{y}=v(x, z)=0 \quad u_{z}=w(x, z)$

Where $\mathrm{u}_{\mathrm{x}}=\mathrm{u}, \mathrm{u}_{\mathrm{y}},=\mathrm{v}$ and $\mathrm{u}_{\mathrm{z}}=\mathrm{w}$ are the displacement components in the $x, y$ and $z$ coordinate axes respectively.

The stress field in the soil is given by the Airy's stress function $\phi(x, z)$ defined by

$$
\sigma_{x x}=\frac{\partial^{2} \phi}{\partial z^{2}} \quad \sigma_{z z}=\frac{\partial^{2} \phi}{\partial x^{2}} \quad \tau_{x z}=-\frac{\partial^{2} \phi}{\partial x \partial z}
$$

Where $\phi$ satisfies the biharmonic equation on the $X Z$ coordinate plane

$$
\nabla^{4} \phi(x, z)=\frac{\partial^{4} \phi}{\partial x^{4}}+2 \frac{\partial^{4} \phi}{\partial x^{2} \partial z^{2}}+\frac{\partial^{4} \phi}{\partial z^{4}}
$$

We apply the exponential Fourier transform method to solve the biharmonic equation for $\phi(x, z)$. Applying the exponential Fourier transform with respect to the space variable $x$, to the biharmonic equation, we have

$\frac{1}{\sqrt{2 \pi}} \int_{-\infty}^{\infty}\left(\frac{\partial^{4} \phi}{\partial x^{4}}+2 \frac{\partial^{4} \phi}{\partial x^{2} \partial z^{2}}+\frac{\partial^{4} \phi}{\partial z^{4}}\right) \exp (-\mathrm{i} \lambda \mathrm{x}) \mathrm{dx}=0$

where $\lambda$ is the Fourier transform parameter and $i=\sqrt{-1}$ is the imaginary number. By the linearity property of the exponential Fourier transform, we obtain: 


$$
\begin{gathered}
\frac{1}{\sqrt{2 \pi}} \int_{-\infty}^{\infty} \frac{\partial^{4} \phi}{\partial x^{4}} \mathrm{e}^{-\mathrm{i} \lambda \mathrm{x}} d x+\frac{1}{\sqrt{2 \pi}} \int_{-\infty}^{\infty} 2 \frac{\partial^{4} \phi}{\partial x^{2} \partial \mathrm{z}^{2}} \mathrm{e}^{-\mathrm{i} \lambda \mathrm{x}} d x \\
+\frac{1}{\sqrt{2 \pi}} \int_{-\infty}^{\infty} \frac{\partial^{4} \phi}{\partial z^{4}} \mathrm{e}^{-\mathrm{i} \lambda \mathrm{x}} d x=0
\end{gathered}
$$

Simplifying Equation (35) becomes:

$$
\begin{aligned}
\frac{1}{\sqrt{2 \pi}}(-i \lambda) \int_{-\infty}^{\infty} \phi(\mathrm{x}, \mathrm{z}) \mathrm{e}^{-\mathrm{i} \lambda \mathrm{x}} d x & \\
& +\frac{1}{\sqrt{2 \pi}} 2(-i \lambda)^{2} \frac{\partial^{2}}{\partial z^{2}} \int_{-\infty}^{\infty} \phi(\mathrm{x}, \mathrm{z}) \mathrm{e}^{-\mathrm{i} \lambda \mathrm{x}} d x \\
& +\frac{1}{\sqrt{2 \pi}} \frac{\partial^{4}}{\partial z^{4}} \int_{-\infty}^{\infty} \phi(\mathrm{x}, \mathrm{z}) \mathrm{e}^{-\mathrm{i} \lambda \mathrm{x}} d x=0
\end{aligned}
$$

Let $\phi(\lambda, z)=\frac{1}{\sqrt{2 \pi}} \int_{-\infty}^{\infty} \phi(\mathrm{x}, \mathrm{z}) \mathrm{e}^{-\mathrm{i} \lambda \mathrm{x}} d x$

Where $\Phi(\lambda, z)$ is the exponential Fourier transform of the Airy's stress function $\phi(x, z)$, then we obtain Equation (38).

$$
\frac{d^{4} \Phi}{d z^{4}}-2 \lambda^{2} \frac{d^{2} \Phi}{d z^{2}}+\lambda^{4} \Phi=0
$$

Thus Equation (38) is a fourth order ordinary differential equation in $\Phi(\lambda, z)$.

We solve using the method of auxiliary (characteristic) polynomials to obtain the characteristic polynomial as

$$
m^{4}-2 \lambda^{2} m^{2}+\lambda^{4}=0
$$

where $\Phi(\lambda, z)=\exp m z$

The roots are

$$
\begin{aligned}
& \left(m^{2}-\lambda^{2}\right)^{2}=0 \\
& m^{2}-\lambda^{2}=0 \text { twice }
\end{aligned}
$$

Hence,

$$
\begin{aligned}
\Phi(\lambda, z)=\left(c_{1}+\right. & \left.c_{2} z\right) \exp (-|\lambda| z) \\
& +\left(c_{3+} c_{4} z\right) \exp (|\lambda| z)
\end{aligned}
$$

where $c_{1}, c_{2}, c_{3}$ and $c_{4}$ are the four constants of integration. For bounded solutions of $\Phi(\lambda, z)$ as $z \rightarrow \infty$, we have $c_{3}=c_{4}=0$.

Hence,

$$
\Phi(\lambda, \mathrm{z})=\left(\mathrm{c}_{1}+\mathrm{c}_{2} \mathrm{z}\right) \exp (-|\lambda| \mathrm{z})
$$

By inversion we obtain, the solution for $\phi(z, x)$ in the domain variables as:

$$
\begin{aligned}
\Phi(\mathrm{x}, \mathrm{z})=\frac{1}{\sqrt{2 \pi}} \int_{-\infty}^{\infty}\left(\mathrm{c}_{1}\right. \\
\left.\quad+\mathrm{c}_{2} \mathrm{z}\right) \exp (-|\lambda| \mathrm{z}) \exp i \lambda x d \lambda \\
\Phi(\mathrm{x}, \mathrm{z})=\frac{1}{\sqrt{2 \pi}} \int_{-\infty}^{\infty}\left(\mathrm{c}_{1}+\mathrm{c}_{2} \mathrm{z}\right) e^{-|\lambda| z} e^{i \lambda x} d \lambda
\end{aligned}
$$

From the Airy stress functions

$$
\begin{aligned}
& \sigma_{z z}=\frac{\partial^{2} \phi}{\partial x^{2}}=\frac{\partial^{2}}{\partial x^{2}} \frac{1}{\sqrt{2 \pi}} \int_{-\infty}^{\infty}\left(\mathrm{c}_{1}\right. \\
& \left.+c_{2} z\right) \exp (-|\lambda| z) \exp (i \lambda x) d \lambda \\
& =\frac{-1}{2 \pi} \int_{-\infty}^{\infty} \lambda^{2}\left(c_{1}+c_{2} z\right) \exp (-|\lambda| z) \exp (i \lambda x) d \lambda \\
& \sigma_{x x}=\frac{\partial^{2} \phi}{\partial x^{2}}=\frac{\partial^{2}}{\partial z^{2}} \frac{1}{\sqrt{2 \pi}} \int_{-\infty}^{\infty}\left(\mathrm{c}_{1}\right. \\
& \left.+c_{2} z\right) \exp (-|\lambda| z) \exp (i \lambda x) d \lambda \\
& =\frac{1}{\sqrt{2 \pi}} \int_{-\infty}^{\infty}|\lambda|\left[|\lambda| c_{1}\right. \\
& \left.+c_{2}(|\lambda| z-2)\right] \exp (-|\lambda| z) \exp (i \lambda x) d x \\
& \tau_{x y}=\frac{-\partial^{2} \phi}{\partial x \partial z}=-\frac{\partial^{2}}{\partial x \partial z} \frac{1}{\sqrt{2 \pi}} \int_{-\infty}^{\infty}\left(c_{1}\right. \\
& \left.+\mathrm{c}_{2} \mathrm{z}\right) \exp (-|\lambda| \mathrm{z}) \exp (i \lambda x) d \lambda \\
& =\frac{1}{\sqrt{2 \pi}} \int_{-\infty}^{\infty} i \lambda\left[|\lambda| c_{1}\right. \\
& \left.+c_{2}(1-|\lambda| z)\right] \exp (-|\lambda| z) \exp (i \lambda x) d x
\end{aligned}
$$

When stresses are given on the $x y$ coordinate plane $(z$ $=0)$, we let the stress boundary conditions to be

$$
\begin{gathered}
\sigma_{z z}(x, 0)=f_{1}(x) \\
\tau_{x y}(x, 0)=f_{2}(x)
\end{gathered}
$$

In (52) and (53), $f_{1}(x)$ and $f_{2}(x)$ are known functions of $x$. Then the exponential Fourier transforms of the stress boundary conditions become:

$$
\begin{gathered}
\bar{\sigma}_{z z}(\lambda, 0)=\bar{f}_{2}(\lambda)=\frac{1}{\sqrt{2 \pi}} \int_{-\infty}^{\infty} f_{1}(x) \exp (-i \lambda x) d x \\
\tau_{z x}(\lambda, 0)=\bar{f}_{1}(\lambda)=\frac{1}{\sqrt{2 \pi}} \int_{-\infty}^{\infty} f_{2}(x) \exp (-i \lambda x) d x
\end{gathered}
$$

By inversion,

$$
\begin{gathered}
\sigma_{z z}(x, 0)=\bar{f}_{1}(\lambda)=\frac{1}{\sqrt{2 \pi}} \int_{-\infty}^{\infty} \bar{f}_{1}(\lambda) \exp i \lambda x d \lambda \\
\tau_{z x}(x, 0)=f_{2}(x)=\frac{1}{\sqrt{2 \pi}} \int_{-\infty}^{\infty} \bar{f}_{2}(\lambda) \exp (i \lambda x) d \lambda
\end{gathered}
$$

From Equations (29) and (31) we have:

$$
\begin{gathered}
\sigma_{z z}(x, 0)=\frac{-1}{\sqrt{2 \pi}} \int_{-\infty}^{\infty} \lambda^{2} c_{1} \exp i \lambda x d \lambda \\
\sigma_{x z}(x, 0)=\frac{-1}{\sqrt{2 \pi}} \int_{-\infty}^{\infty} i \lambda\left[|\lambda| c_{1}-c_{2}\right] \exp i \lambda x d \lambda
\end{gathered}
$$

Comparing Equations (56) and (58) and Equations (57)and (59) we obtain:

$$
-\lambda^{2} c_{1}=\bar{f}_{1}(\lambda)
$$




$$
i \lambda\left(|\lambda| c_{1}-c_{2}\right)=\bar{f}_{2}(\lambda)
$$

Solving,

$$
\begin{gathered}
c_{1}=\frac{-f_{1}(\lambda)}{\lambda^{2}} \\
i \lambda\left(|\lambda| c_{1}-c_{2}\right)=f_{2}(\lambda) \\
|\lambda| c_{1}-c_{2}=\frac{f_{2}(\lambda)}{i \lambda} \\
c_{2}=|\lambda| c_{1}-c_{2}=\frac{f_{2}(\lambda)}{i \lambda} \\
c_{2}=-|\lambda| \frac{f_{1}(\lambda)}{\lambda^{2}}-\frac{f_{2}(\lambda)}{i \lambda} \\
c_{2}=-|\lambda|\left(\frac{f_{1}(\lambda)}{\lambda^{2}}+\frac{f_{2}(\lambda)}{i \lambda}\right)
\end{gathered}
$$

Hence,

$$
\begin{aligned}
& \sigma_{z z} \\
& =\frac{1}{\sqrt{2 \pi}} \int_{-\infty}^{\infty}(-i \lambda z) \bar{f}_{2}(\lambda) \\
& +(1+z|\lambda|) \bar{f}_{1}(\lambda) \exp (-|\lambda| \mathrm{z}) \exp i \lambda x d \lambda \\
& \sigma_{x x} \\
& =\frac{1}{\sqrt{2 \pi}} \int_{-\infty}^{\infty}\left[\left(i \lambda-\frac{z|\lambda|}{\lambda}\right) \bar{f}_{2}(\lambda)\right. \\
& \left.+(1+z|\lambda|) \bar{f}_{1}(\lambda)\right] \exp (-|\lambda| \mathrm{z}) \exp i \lambda x d \lambda \\
& \tau_{x z} \\
& =\frac{1}{\sqrt{2 \pi}} \int_{-\infty}^{-\infty}\left[-z \lambda f_{2}(\lambda)\right. \\
& \left.+(i z \lambda) f_{1}(\lambda)\right] \exp (-|\lambda| z) \exp i \lambda x d \lambda
\end{aligned}
$$

We now consider the case of a uniformly distributed normal line load $p$ per unit length acting on the $y$ axis (at $x=0$ ). Then:

$$
\begin{aligned}
& f_{1}(x)=+P \delta(y)=+P \delta(x-0) \\
& f_{2}(x)=0
\end{aligned}
$$

Where $\delta$ is the Dirac delta function.

Hence,

$$
\begin{aligned}
& \bar{f}_{1}(\lambda)=\frac{+P}{\sqrt{2 \pi}} \\
& \bar{f}_{2}(\lambda)=0
\end{aligned}
$$

Then:

$$
\begin{gathered}
c_{1}=\frac{-f_{1}(\lambda)}{\lambda^{2}}=\frac{-P}{\sqrt{2 \pi}} \frac{1}{\lambda^{2}} \\
c_{2}=-|\lambda| \frac{f_{1}(\lambda)}{\lambda^{2}}=\frac{|\lambda|}{\sqrt{2 \pi}} \frac{P}{\lambda^{2}}
\end{gathered}
$$

Then:

$$
\begin{gathered}
\sigma_{z z}(x, z)=\frac{p}{2 \pi} \int_{-\infty}^{\infty}(1+z|\lambda|) e^{-|\lambda|} e^{i \lambda x} d \lambda \\
=\frac{P}{2 \pi}\left\{\int_{-\infty}^{\infty} e^{-|\lambda| z} e^{i \lambda x} d \lambda+z \int_{-\infty}^{\infty}|\lambda| e^{-|\lambda| z} e^{i \lambda x} d \lambda\right\}
\end{gathered}
$$

$$
\begin{gathered}
=\frac{p}{2 \pi}+\left\{\frac{2 z}{x^{2}+z^{2}}+\frac{2 z\left(z^{2}-x^{2}\right)}{\left(z^{2}+x^{2}\right)^{2}}\right\} \\
=\frac{p}{2 \pi}\left(\frac{4 z^{3}}{\left(x^{2}+z\right)^{2}}\right)=\frac{2 p}{\pi}\left(\frac{z^{3}}{\left(z^{3}+x\right)^{2}}\right) \\
\sigma_{x x}(x, z) \\
=\frac{p}{2 \pi} \int_{-\infty}^{\infty}(1-z|\lambda|) \exp (-|\lambda| z) \exp i \lambda x d \lambda \\
=\frac{p}{2 \pi}\left(\frac{2 z}{x^{2}+z^{2}}-\frac{2 z\left(z^{2}-x^{2}\right)}{\left(z^{2}+x^{2}\right)^{2}}\right) \\
=\frac{2 p}{\pi}\left(\frac{z x^{2}}{\left(x^{2}+z^{2}\right)^{2}}\right) \\
\tau_{x z}(z, x)=\frac{p}{2 \pi} \int_{-\infty}^{\infty} i z \lambda \exp (-|\lambda| z) \exp i \lambda x d \lambda \\
=\frac{2 p}{\pi}\left(\frac{z^{2} x}{\left(x^{2}+z^{2}\right)^{2}}\right)
\end{gathered}
$$

An interesting application of the line load solution is in determining the elastic lateral stress distribution on a rigid retaining wall due to a line load applied parallel to the wall on the backfill surface as shown in Figure 6.

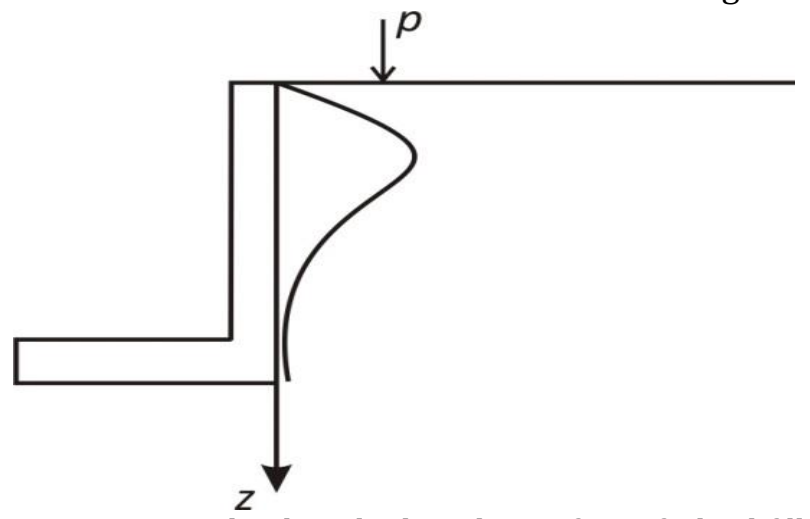

Figure 4: Line load applied on the surface of a backfill supported by a wall

By considering the problem of two line loads of equal intensity acting at $x=+a$ and $x=-a$, on an elastic half space soil and the conditions on the axis of symmetry $x=0$, the distribution of horizontal stress on the rigid wall is given from Flamant's solution derived in this study using the Fourier transform method by:

$$
\sigma_{x x}(z)=\frac{4}{\pi} \frac{P a^{2} z}{\left(a^{2}+z^{2}\right)^{2}}
$$

The maximum value of $\sigma_{x x}$ occurs at $z=0.577 a$, and the maximum value is:

$$
\sigma_{\text {xxmax }}=0.4135 \frac{p}{a}
$$

The resultant lateral force is: 


$$
\begin{gathered}
P_{e}=\int_{a}^{H} \sigma_{x x}(z) d z \\
P_{e}=\frac{2}{\pi} \frac{P}{1+a^{2} / H^{2}} \\
\text { If } a=0, p_{e}=0.637 p
\end{gathered}
$$

Table 1 shows the distribution of $\Delta \sigma_{V}$ with depth $z$ and obtained in this study and comparison with the solution by Das.

Table 1: Variation of $\sigma_{v} /(q / z)$ with $x / z$

\begin{tabular}{ccc}
\hline$x / z$ & $($ Das, $[2]) \frac{\sigma_{Z Z}}{q / z}$ & Present study \\
\hline 0 & 0.637 & 0.637 \\
0.1 & 0.624 & 0.624 \\
0.2 & 0.589 & 0.589 \\
0.3 & 0.536 & 0.536 \\
0.4 & 0.473 & 0.473 \\
0.5 & 0.407 & 0.407 \\
0.6 & 0.344 & 0.344 \\
0.7 & 0.287 & 0.287 \\
0.8 & 0.237 & 0.237 \\
0.9 & 0.194 & 0.194 \\
1.0 & 0.159 & 0.159 \\
1.5 & 0.060 & 0.060 \\
2.0 & 0.025 & 0.025 \\
3.0 & 0.006 & 0.006 \\
\hline
\end{tabular}

\section{DISCUSSIONS AND CONCLUSIONS}

The stresses induced in a semi-infinite linear elastic soil due to an infinitely long line load of intensity $p$ has been obtained using Airy's stress function and the exponential Fourier transform method. The stresses obtained were found to be exactly identical with classical solutions obtained by Flamant. The stresses determined in this work were also identical with solutions found in the technical literature obtained by integrating Boussinesq's solution for a point load. The effectiveness and accuracy of the exponential Fourier transform technique is thus illustrated. The stresses from the infinite line load were observed to be effectively distributed in only one horizontal direction, as opposed to two for the point load. The vertical stresses under the line of application of the load decrease with the first power of the depth, and at any depth, the vertical stresses are distributed more widely than for the case of point load. It was observed further that the vertical stress distribution $\sigma_{z z}$ is independent of the Poisson's ratio, $\mu$.
The distribution of stresses $\sigma_{X x}, \sigma_{z z}$ and $\tau_{X z}$ determined in this study are applied in evaluating the response of a soil to a surface loading by a long narrow footing, which can be idealized as an infinitely long line loading on a semi-infinite elastic soil. The expressions are simpler to use than those expressions for rectangular strip loads.

\section{REFERENCES}

[1] Timoshenko, S.. Theory of Elasticity. McGraw Hill Book Co. New York. Pp. 338 - 339. 1948.

[2] Das, B. M. Advanced Soil Mechanics Third Edition, Taylor and Francis. London and New York. 2008.

[3] Verruijt, A. Soil Mechanics, Delft University of Technology. 2006.

[4] Richards, R. Principles of Solid Mechanics. CRC Press, Washington D. C. 2001.

[5] Cummings, A. E. Distribution of Stresses under a Foundation, Transactions. American Society of Civil Engineers Vol. 101 pp. 1072 - 1134. 1936.

[6] Gray, H.. Stress Distribution in Elastic Solids. Proceedings International Conference on Soil Mechanics and Foundation Engineering Vol. 2 pp 157 - 168. Cambridge. 1936.

[7] Timoshenko, S. and Goodier, J. N.. Theory of Elasticity. McGraw Hill Book Co. Inc. New York. 1951.

[8] Newmark, N.M. Stress Distribution in Soils Proceedings Purdue Conference on Soil Mechanics and its Applications pp 295 - 303. 1940.

[9] Holl, D.L. Plane Strain Distribution of Stress in Elastic Media. Iowa Engineering Experiment Station Bull 148 55pp. 1941.

[10] Foster, C. R. and Fergus, S. M. Stress Distribution in a Homogeneous Soil. Research Report No. 12 - F. Highway Research Board pp 36. 1951.

[11] Raj, P. P. Soil Mechanics and Foundation Engineering, Pearson Education, London. 2011.

[12] Hetnarski, R. B. and Ignaczak, J. The Mathematical Theory of Elasticity, Second Edition. CRC Press Taylor and Francis Group, New York. 2012.

[13] Olson, R. E. Advanced Soil Mechanics. Department of Construction Engineering, Chaoyang University of Technology. 1989. 